\title{
The Absence of Tunnel Sensations in Near-Death Experiences from India
}

\author{
Allan Kellehear, Ph.D. \\ La Trobe University
}

Ian Stevenson, M.D.

University of Virginia

Satwant Pasricha, Ph.D.

National Institute of Mental Health and

Neurosciences, India

Emily Cook, Ph.D.

University of Virginia

ABSTRACT: This article questions the recent report by Susan Blackmore (1993) of tunnel sensations in near-death experiences in India, and presents anthropological and methodological reasons for doubting the validity of that finding.

\footnotetext{
Allan Kellehear, Ph.D., is Senior Lecturer in Sociology at La Trobe University in Bundoora, Australia; Ian Stevenson, M.D., is Carlson Professor of Psychiatry at the University of Virginia; Satwant Pasricha, Ph.D., is Additional Professor of Clinical Psychology at the National Institute of Mental Health and Neurosciences in Bangalore, India; and Emily Cook, Ph.D., is Research Assistant Professor of Psychiatry at the University of Virginia.

Dr. Stevenson's research receives support from the Bernstein Brothers Parapsychology and Health Foundation and from the Nagamasa Azuma Fund of the University of Virginia; Dr. Pasricha acknowledges the support of the National Institute of Mental Health and Neurosciences in India. Reprint requests should be addressed to Dr. Kellehear at the School of Sociology and Anthropology, La Trobe University, Bundoora, Victoria 3083, Australia.
} 
In this paper we comment on several issues raised by Susan Blackmore's (1993) recent research into near-death experiences (NDEs) in India. Blackmore solicited, by advertisement in an Indian newspaper, reports of "people who had come close to death." Of the 19 reports that she received in response to this advertisement, only eight were considered NDEs. Of these eight reports, three respondents reported sensations of darkness. One of these respondents accepted a descriptor ("tunnel") for this darkness that was offered by Blackmore in a follow-up survey. On the basis of these data, Blackmore concluded that the tunnel sensation in NDEs appears independent of cultural influences. In this connection, those findings are also interpreted as support for her neurophysiological theory of tunnel sensations.

Our argument is that the theoretical and methodological problems in Blackmore's work are too serious to warrant the conclusions she drew. We will identify both general problems and specific issues that have equal bearing on Blackmore's research design and conclusions.

First, the conclusion that the tunnel sensation appears independent of a person's culture is not warranted from any single case study. This is an overgeneralized conclusion. No single case study, whether from India or from Japan, can indicate broad cross-cultural patterns of human experience. Quite clearly a broader cross-cultural examination of NDEs involving several distinctly different cultures is needed for any generalization such as this. In this connection, it is also not clear, other than in the most general way, that India has a culture structurally different from European cultures. This brings us to our second point.

Even if some persons in India have the tunnel experience, this would not indicate its universality, since both Indian and European cultures are dominated by historical religions. Historical religions, such as Christianity, Islam, or Hinduism, tend to occur in cultures of long-term settlement, where tunnels are common technological and architectural forms. In societies influenced by primitive religions, such as hunter-gatherer communities, tunnels may not be of common occurrence and hence cultural significance. The evidence of this argument, such as it is, has been discussed by Allan Kellehear (1993).

Third, despite the theoretical possibility that people from India may experience a tunnel sensation in their NDEs, recent work by Satwant Pasricha $(1992,1993)$ has failed to uncover this so far. Pasricha has been conducting a survey of NDEs in south India. Her reports show that the cases there are closely similar, with some differences, to those of north India previously reported by Pasricha and 
Ian Stevenson (1986). Cases investigated in India now number altogether 45, and not a single informant has reported the experience of a tunnel. Moreover, these are cases investigated by interviews with the informants, not with correspondence through the mail.

Fourth, all the above comments notwithstanding, an inductive analysis of the material presented by Blackmore does not indicate that tunnels are an important feature of the NDEs in her sample from India. On the contrary, all three respondents in this category merely reported (spontaneously) sensations of darkness. Only by asking a leading question employing the term "tunnel" did Blackmore obtain an assent from one of the respondents to this particular symbol for the darkness. If anything, this might lend support to the idea of the tunnel as one descriptor among others that might be offered and accepted by those attempting to describe great darkness. In this connection, we also note that Blackmore used leading questions in inquiring about the light. Two of her questions assumed that the subjects had experienced lights. Aside from the fact that the data did not suggest a tunnel sensation, the reports, such as they are, did not even support Blackmore's own theory of tunnels.

According to Blackmore, random firing of cortical neurons should "produce a much brighter impression in the center of the field of view, fading out toward the periphery: in other words, a tunnel pattern" (1993, p. 207). However, that prediction was not supported by her data. Of the eight people considered to have undergone NDEs, two had no experiences of light or darkness; three experienced light alone; two experienced only darkness; and one person reported alternating experiences of light and darkness. Of the three people who were considered by Blackmore to have experienced "tunnels specifically or dark places," these individuals did not exhibit the hypothesized features of darkness. In other words, no respondents reported a bright impression in the center of their visual field.

The above points notwithstanding, Blackmore exaggerated the importance of these data by attaching percentage labels to single digit sample sizes. By lumping a single (and questionable) case of "tunnel" sensation with two other cases in which the subjects reported an experience of darkness, she permitted herself the claim that 38 percent of her respondents could be counted as having had the tunnel experience. We direct readers' attention to a percentage figure (38) that is over twelve times the actual sample number (3)! Considering that the effective size of this sample is eight persons, we consider the claim of 38 percent (representing the dubious conflation of three 
somewhat different reports) to be an example of torturing data until they give you the answer you need (Mills, 1993).

Fifth, although Blackmore acknowledged that the readers of the Times of India are "not at all representative of Indians in general" (1993, p. 215), she discussed her results as if they were representative. They most certainly are not. The Times of India is read only by a highly educated and English-speaking readership. If Blackmore had wished to sample more widely, and more typically, she might have arranged for her notice to be published in a Hindi-medium newspaper, such as the Nav Bharat. The cases investigated by Pasricha and Stevenson (1986) and by Pasricha (1992, 1993) came from the heart of India, not from the Westernized readers of the Times of India.

We consider it unfortunate that Blackmore has adopted as paradigmatic the features of the NDE that Raymond Moody (1975) described in his first book. That was a popular book, and Moody has never claimed anything else for it. He told one of us (I.S.) that he deliberately did not try to provide figures of the incidence of NDE features, but included a feature in his list even when only a few persons mentioned it to him. In fact, the tunnel experience is far from universal even among Western cases. Kenneth Ring (1980) found that only 23 percent of his subjects had the experience of darkness, and only a minority of these described passage through a tunnel. Bruce Greyson and Stevenson (1980) found the tunnel experience reported by only 31 percent of 78 subjects. These low incidences are insufficient to support a neurophysiological interpretation of NDEs because such a theory leaves unexplained why so many persons even in the West do not have the tunnel experience.

Finally, Blackmore's strategy of developing biological theories for the NDE before examining the cultural prevalence of their phenomenology reflects both a strangely backward set of priorities and an overconfidence in what the cross-cultural material might yield. Such optimism can lead, as it appears to have done in this case, to leading questions and overgeneralization.

\section{Reference}

Blackmore, S. J. (1993). Near-death experiences in India: They have tunnels too. Journal of Near-Death Studies, 11, 205-217

Greyson, B., and Stevenson, I. (1980). The phenomenology of near-death experiences. American Journal of Psychiatry, 137, 1193-1196. 
Kellehear, A. (1993). Culture, biology, and the near-death experience: A reappraisal. Journal of Nervous and Mental Disease, 181, 148-156.

Mills, J. L. (1993). Data torturing. New England Journal of Medicine, 329, 1196-1199. Moody, R. A., Jr. (1975). Life after life. Covington, GA: Mockingbird Books.

Pasricha, S. (1992). Near-death experiences in south India: A systematic survey in Channapatna. National Institute of Mental Health and Neuro Sciences Journal, 10 , 111-118.

Pasricha, S. (1993). A systematic survey of near-death experiences in south India. Journal of Scientific Exploration, 7, 161-171.

Pasricha, S., and Stevenson, I. (1986). Near-death experiences in India: A preliminary report. Journal of Nervous and Mental Disease, 174, 165-170.

Ring, K. (1980). Life at death: A scientific investigation of the near-death experience. New York, NY: Coward, McCann and Geoghegan. 